may represent a novel biomarker of arrhythmic risk. Sarcomeric mutation status independently predicts a blunted stress oxygenation response in HCM.

\section{PREVALENCE AND CORRELATES OF MID-WALL LATE GADOLINIUM ENHANCEMENT IN PATIENTS WITH ISCHEMIC CARDIOMYOPATHY}

Zoi Tsoumani, Chris Miller, Matthias Schmitt, Gaetano Nucifora. North-West Heart Centre, Manchester University NHS Foundation Trust

10.1136/heartjnl-2019-BSCMR.23

Background Late gadolinium enhancement (LGE) imaging is an established technique for the assessment of myocardial replacement fibrosis/scar. The presence of mid-wall LGE has been described in $\sim 30 \%$ of patients with dilated cardiomyopathy (DCM) of non-ischemic aetiology and is known to be associated with poor clinical response to pharmacological therapy and worse outcome. Conversely, little is known regarding the clinical significance of additional mid-wall LGE in patients with ischemic cardiomyopathy (ICM). The aim of the present study was therefore to investigate the prevalence and clinical/ imaging correlates of mid-wall LGE in a consecutive cohort of patients with ICM.

Methods The UHSM-redCAP database was searched for patients with ICM (defined as the presence of LV ejection fraction $<50 \%$ in the context of multivessel disease and/or previous myocardial infarction) who had clinically-indicated CMR with LGE imaging for the assessment of LV volumes, LV regional/global systolic function and presence/extent of myocardial replacement fibrosis/scar.

Results A total of 606 consecutive patients with ICM were identified and included in the study. Mean age of the study population was $64 \pm 11$ years and $85 \%$ of the patients were males. Mid-wall LGE was observed in 34 (6\%) patients. No significant difference in age (64 \pm 11 vs. $65 \pm 11$ years), male gender (85\% vs. 94\%), and prevalence of NYHA functional class 3-4 (29\% vs. 32\%) was observed between ICM patients without vs. with additional mid-wall LGE.
However, ICM patients with mid-wall LGE had larger LV end-diastolic volume $(261 \pm 79 \mathrm{ml}$ vs. $220 \pm 60 \mathrm{ml} ; \mathrm{p}<0.001)$, larger LV end-systolic volume $(176 \pm 74 \mathrm{ml}$ vs. $140 \pm 54 \mathrm{ml}$; $\mathrm{p}<0.001)$ and lower LV ejection fraction $(34 \% \pm 8 \%$ vs. 38 $\pm 9 \% ; \mathrm{p}=0.019$ ).

Conclusion Additional mid-wall LGE is present in a small proportion $(6 \%)$ of patients with ICM and is a marker of worse LV dilatation and systolic function. The clinical meaning of this finding (i.e. dual pathology vs. epiphenomenon of severe LV dilatation and wall stress) and its implication with respect to outcome remains to be determined.

Funding acknowledgement This research was funded by the NIHR Leicester Cardiovascular Biomedical Research Centre.

\section{ADENOSINE STRESS NATIVE T1 MAPPING DEMONSTRATES IMPAIRED MYOCARDIAL PERFUSION RESERVE IN NON-ISCHEMIC DILATED CARDIOMYOPATHY}

Zoi Tsoumani, Chris Miller, Matthias Schmitt, Gaetano Nucifora. North-West Heart Centre, Manchester University NHS Foundation Trust

\subsection{6/heartjnl-2019-BSCMR.24}

Background Impaired myocardial perfusion reserve has been demonstrated in non-ischemic dilated cardiomyopathy (NIDCM) by positron emission tomography (PET) and adenosine-stress first-pass perfusion cardiac magnetic resonance (CMR) imaging. Adenosine stress native T1 mapping is a novel CMR technique able to assess myocardial perfusion without the use of contrast agents. Aim of the present study was to the determine the clinical utility of this novel CMR technique in NIDCM.

Methods A total of 20 consecutive patients (mean age $61 \pm 12$ years, $80 \%$ males) with diagnosis of NIDCM who consented to be enrolled in the UHSM CMR registry were included in the present study. CMR at $3 \mathrm{~T}$ including 1 . cine imaging for the assessment of LV volumes, mass and global longitudinal strain (GLS) by tissue-tracking imaging; 2. rest and stress (adenosine $140 \mathrm{mcg} / \mathrm{kg} / \mathrm{min}$ ) MOLLI T1 mapping of mid

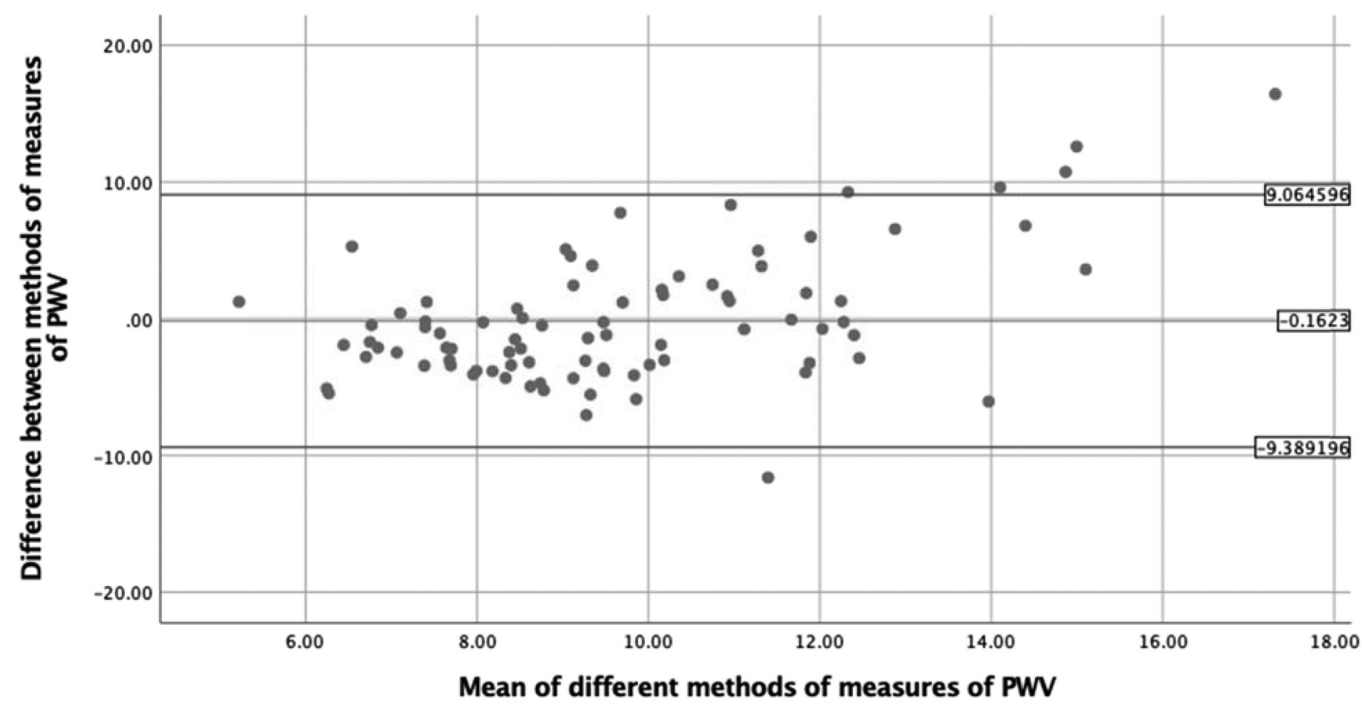

Abstract 23 Figure 1 Bland Altman Plot comparing phase contrast MRI measured PWV and cfPWV measured by oscillometry. Parametres used: Mean of difference $=-0.1623, S D \pm 4.70760$; LoA: lower limit $=-9.389196$, upper limit $=9.064596$ 


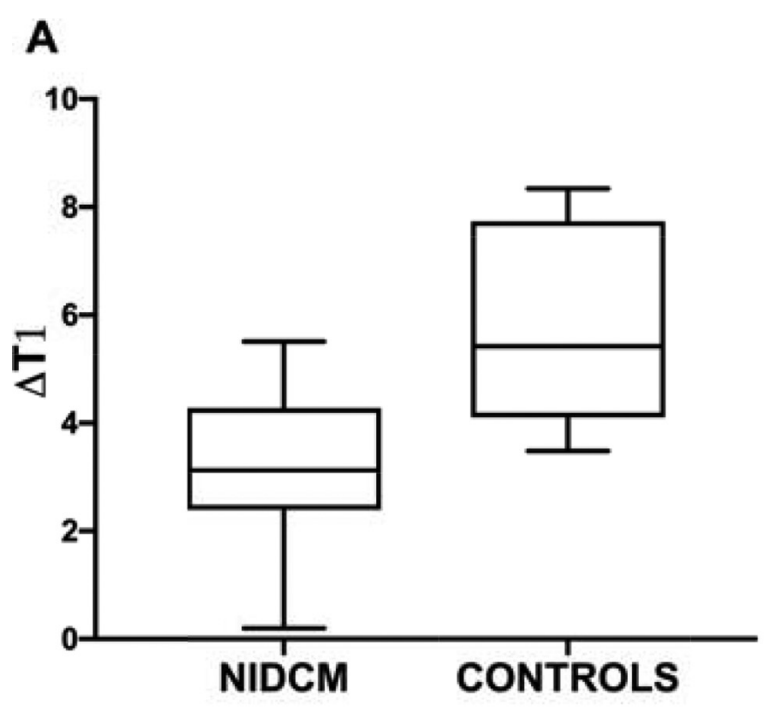

B

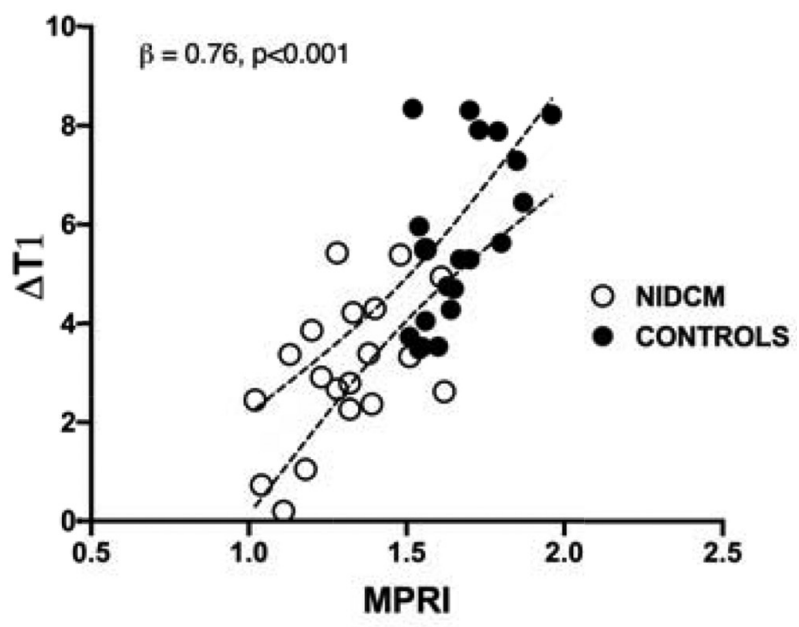

Abstract 24 Figure 1

ventricular slice for the assessment of rest and stress $\mathrm{T} 1$ values and $\mathrm{T} 1$ reactivity $(\Delta \mathrm{T} 1 \%) ; 3$. first-pass perfusion imaging for the assessment of myocardial perfusion reserve index (MPRI) and 4. late gadolinium enhancement (LGE) imaging for the assessment of myocardial replacement fibrosis, was performed. Twenty control patients without history of known coronary artery disease and evidence of reversible ischemia or previous myocardial infarct on CMR imaging were included for comparison purposes.

Results NIDCM patients had significantly higher native T1 value $(1263 \pm 47 \mathrm{~ms}$ vs. $1234 \pm 38 \mathrm{~ms}, \mathrm{p}=0.031)$, significantly lower $\Delta \mathrm{T} 1 \% \quad(3.2 \% \pm 1.5 \%$ vs. $5.7 \pm 1.7 \%, \quad \mathrm{p}<0.001$, figure $1 \mathrm{~A})$, significantly lower MPRI $(1.32 \pm 0.18$ vs. $1.67 \pm 0.13$, $\mathrm{p}<0.001)$ and significantly lower GLS $(-10 \pm 4 \%$ vs. -16 $\pm 2 \%, \mathrm{p}<0.001)$ as compared to controls. A significant strong relation between $\Delta \mathrm{T} 1 \%$ and MPRI $(B=0.76, p<0.001$, figure $1 \mathrm{~B})$ and a significant moderate relation between DT1\% and GLS $(ß=-0.54, \mathrm{p}<0.001)$ were observed.

Conclusion T1 reactivity, myocardial perfusion reserve and GLS are significantly reduced in NIDCM patients compared to controls. Adenosine stress T1 mapping holds promise for detection of impaired myocardial perfusion reserve in NIDCM without requirement for contrast agents.

\section{MITRAL INFLOW VELOCITY ENCODED IMAGING BY CMR FOR THE ASSESSMENT OF LEFT VENTRICULAR HAEMODYNAMICS}

${ }^{1}$ Nithin Balasubramanian, ${ }^{1}$ Natasha Barker, ${ }^{1}$ Benjamin Fidock, ${ }^{1}$ Alistair W Macdonald, ${ }^{1}$ David Capener, ${ }^{1}$ Christopher S Johns, ${ }^{2}$ Kavitasagary Karunasaagarar, ${ }^{2}$ Graham Fent,

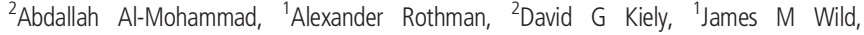
${ }^{1}$ Andrew Swift, ${ }^{1}$ Pankaj Garg. 'Department of Infection, Immunity and Cardiovascular disease, University of Sheffield, Sheffield, UK; ${ }^{2}$ Sheffield Teaching Hospitals NHS Foundation Trust, Sheffield, UK

\subsection{6/heartjnl-2019-BSCMR.25}

Background Heart failure is common and is associated with high mortality and morbidity. Haemodynamic assessment is critical to diagnosis and therapeutic monitoring. Non-invasive Doppler echocardiography is commonly used to assess peak early and late mitral inflow velocities to predict left ventricular (LV) haemodynamics. Cardiovascular magnetic resonance imaging offers two-dimensional through plane flow assessment using phase contrast acquisition. It remains unclear which through plane mitral inflow velocity (averaged or peak) best predicts LV haemodynamics better.

Purpose The objective of this study is to investigate which through plane mitral inflow velocities (averaged or peak) is better associated with invasive left heart haemodynamic parameters. Methods In this observational study, 16 patients were prospectively recruited at a large tertiary pulmonary hypertension unit. These patients had invasive right heart haemodynamic catheter-based study (RHC). In addition, all patients received CMR on the $1.5 \mathrm{~T}$ scanner (HDx scanner, GE Healthcare, Waukesha, Wisconsin, USA), using an 8-channel cardiac coil. CMR protocol included long and short axis cines and mitral inflow phase contrast acquisition. The LV haemodynamic parameters assessed by RHC included the following: pulmonary capillary wedge pressure (PCWP), cardiac output, cardiac index, systemic vascular resistance (SVR). Cardiac output assessment was done by standard thermodilution method.

Results Mean age of the 16 patients analysed was $68.7 \pm 7.9$ years and $56.3 \%$ were female. Of the 16 patients, 5 patients had non-analysable data. Mean PCWP was $11.1 \pm 4.7 \mathrm{mmHg}$, mean cardiac output was $4.4 \pm 1.3 \mathrm{~L} / \mathrm{min}$, mean cardiac index was $2.4 \pm 0.8 \mathrm{~L} / \mathrm{min} / \mathrm{m}^{2}$ and mean SVR was $1827 \pm 589$ dynes/ seconds $/ \mathrm{cm}^{-5}$. Peak EA ratio correlated with PCWP $(r=0.68$ $\mathrm{p}=0.02)$ and with cardiac index $(\mathrm{r}=0.68 \mathrm{p}=0.02)$. There was a notable correlation between mean EA ratio and cardiac index $(\mathrm{r}=0.66 \mathrm{p}=0.03)$. Mean A showed a significant correlation with SVR $(r=0.64, p=0.03)$.

Conclusions Peak mitral inflow velocities are more closely associated with LV haemodynamics. This may be due to

Abstract 25 Table 1 Mitral inflow 2D flow parameters and their association to left heart haemodynamics

\begin{tabular}{lccccc}
\hline CMR parameters & Mean & SD & \multicolumn{3}{c}{ Pearson Correlation (P-value) } \\
\hline & & & PCWP & Cardiac Index & SVR \\
\hline Peak E velocity (cm/sec) & 55.6 & 29 & $0.41(0.13)$ & $0.22(0.33)$ & $0.28(0.53)$ \\
Peak A velocity (cm/sec) & 52.2 & 19 & $0.30(0.37)$ & $0.24(0.17)$ & $0.37(0.06)$ \\
EA ratio & 1.1 & 0.5 & $0.69(0.02)$ & $0.68(0.02)$ & $0.58(0.07)$ \\
MV DT (milliseconds) & 210 & 71 & $0.32(0.10)$ & $0.29(0.44)$ & $0.34(0.55)$ \\
Mean E (cm/sec) & 13.4 & 6 & $0.21(0.55)$ & $0.14(0.22)$ & $0.17(0.90)$ \\
Mean A (cm/sec) & 8.2 & 4 & $0.44(0.17)$ & $0.56(0.03)$ & $0.64(0.03)$ \\
Mean EA ratio & 2.2 & 2 & $0.41(0.21)$ & $0.66(0.03)$ & $0.44(0.20)$ \\
\hline
\end{tabular}

